Original article

\title{
Role of pharmacist led home medication review in community setting and the preparation of medication list
}

\author{
Dilip Chandrasekhar*, Elizabeth Joseph, Farzana Abdul Ghaffoor, Hridya Mary Thomas \\ Department of Pharmacy Practice, Al Shifa College of Pharmacy, Poonthavanam P.O., Khizhattur, Perinthalmanna, Kerala, 679325, India
}

\section{A R T I C L E I N F O}

\section{Article history:}

Received 27 July 2017

Received in revised form 1 January 2018

Accepted 2 January 2018

Available online 3 January 2018

\section{Keywords:}

Medication errors

General practitioner

Medication regimen

Quality of life

Adverse drug reaction

Medication adherence

\begin{abstract}
A B S T R A C T
Background: Home Medicines Review (HMR) is a patient-focused, meticulous and collaborative health care service provided in the community setting, to enhance quality use of medicines and patient understanding. This service is framed to assist the patients living at home to augment the benefit of their medication regimen and prevent medication related problems.

Objectives: This study was conducted to identify, prevent and resolve potential medication-related problems, optimise pharmacotherapy and assist in achieving better health outcomes for patients at home.

Methods: It was a cross-sectional interventional study conducted for a period of 6 months in 85 patients in different regions of Malappuram district where discrepancies of the prescriptions, knowledge gap of the patients, use of other medication and storage conditions of medicines were evaluated.

Results: Data from a total of 85 patients were collected among which 48 were males (56\%) followed by females $(n=37,44 \%)$ with a mean age group of $40-59 y e a r s(47 \%)$. The patient had a lack of knowledge in factors like the name of the drug (34\%), the reason for taking the medication (27\%), etc. Drug interaction was the main discrepancy found in majority of the prescriptions. Around $32 \%$ of the population experienced ADR on taking the medication and among the patients interviewed, $64 \%$ of them didn't use any OTC drugs along with prescribed drugs. Around 60 of the interviewed patients stored multiple drugs in a same container and 52 of the patient's medicines had illegible labels.

Conclusion: The study clearly showed that the subjects were unaware of the HMR service and yet majority were accepting the program.
\end{abstract}

(C) 2018 Published by Elsevier, a division of RELX India, Pvt. Ltd on behalf of INDIACLEN.

\section{Introduction}

Home Medicines Review (HMR) is a consumer-focused, structured and collaborative health care service provided in the community setting, to optimise quality use of medicines and consumer understanding. ${ }^{1}$ It is a service designed to assist consumers living at home to maximise the benefit of their medication regimen \& prevent medication related problems. Being a patient - focused process it advocates the optimal and quality of medication at the patient's home. It involves systematic assessment of the patient's medication in order to identify and meet the medication related needs as well as to identify, resolve and prevent drug related problems. ${ }^{2}$ This service is a continuity of patient's care from ward to their home.

\footnotetext{
* Corresponding author at: Dilip Chandrasekhar, Dept of Pharmacy Practice, Alshifa College of Pharmacy, Poonthavanam, P.O., Perinthalmanna, Kerala, 679325, India.

E-mail address: dilipc@alshifacollegeofpharmacy.ac.in (D. Chandrasekhar).
}

The patients find it difficult and uneasy to get medical care in conventional settings like hospitals, nursing homes. There are various causes and reasons why patients although aware of the importance of getting the timely health care services, fail to do so. The ignorance of the patients regarding the consequences is an example for it. They are not very much enthusiastic of meeting a doctor and getting evaluated. The patient would prefer to receive the health care in a personalized approach with confidentiality where the others do not come to know regarding his/her illness. In good old days the family doctors used to visit the patients at their home and provide care. The patients felt secure and comfortable of being treated at home. This also avoided hospital ward charges as well as need for an attendant for patients. This style of health care provision vanished due to increased workload and less number of doctors. But now a day the busy schedule of physicians has some limitations in acquiring complete information of the patients. Here comes the role of pharmacists. Through HMR, the patient information is acquired by the pharmacist on referral from the General Practitioner(GP). 
Medication review can be classified into four types: prescription review, adherence support review, clinical review and clinical review with prescribing. A prescription review aims to address the technical issues of a patient's prescription such as anomalies or changed items. An adherence support review, with the patient present, addresses a patient's medication-taking behaviour focusing on improving a patient's knowledge of medicines and adherence to them. A clinical medication review, with access to clinical notes and the patient present, is more comprehensive and addresses the patient's use of medication in the context of their clinical condition. In some countries an extension of type 3 exists and includes the authority for prescribing. These services, the last two particularly, must be conducted in close collaboration with physicians and other health professionals.

The emergence of health conscious society has increased the number of physician visits by the patients. This naturally increased the prescription drug consumers. The overwhelming consciousness also increased the number of physicians visiting at a time. In case of older people, multiple drugs are prescribed which have to be taken at different times of the day, often using multiple dose forms and multiple routes of administration. ${ }^{3}$ Polypharmacy in elderly patient has a high probability of ADR, drug interaction, prescribing cascade and high cost, which is also associated with decreased quality of life, decreased mobility and cognition. The practice of using alternative medicines apart from the conventional medical treatments in a belief of improving the efficacy of the treatment without established scientific evidence, can lead to undesired effect like decreased therapeutic effect of the conventional medicine or an interaction with it. In these cases HMR helps to achieve safe, effective and appropriate use of medicines by detecting and addressing medication-related problems that interfere with desired consumer outcomes. The HMR consists of visits by a pharmacist and analyzes the patients from the point of drug, disease and life style. Here the pharmacist after having a dialogue with the patient, prepares a customized care plan and implements it to the patient by explaining and educating about the disease, drug and lifestyle modifications. He also prepares a medication therapy management plan as per the prescription. The patients are to be visited periodically to assess the outcomes and documents for further treatment. The pharmacist with his skills tries to decipher the reasons for varied or failed outcomes. He can communicate with doctors and nurses regarding the diagnosis and treatment to discuss the patient care issues. HMR encourages patient centric care. ${ }^{4}$

This study was conducted to identify, prevent and resolve actual or potential medication-related problems, optimise pharmacotherapy and assist in achieving better health outcomes for patients at home. The main objectives of this study were to achieve safe, effective and appropriate use of medicines by detecting and addressing medication-related problems that interfere with desired patient outcomes. Improving the patient's quality of life and health outcomes by providing advice on the management of their medicine was anticipated during the study. Improving consumer and health professional knowledge and understanding about medicines would help the patients in achieving better quality of life which expected to be made possible with HMR. The study also proposed to facilitate cooperative working relationships between members of the health care team in the interests of consumer health and well-being; and to provide medicine information to the consumer and other health care providers involved in the consumer's care.

\section{Methodology}

A cross-sectional interventional study was conducted for duration of 6 months among the different regions in Malappuram district. In order to identify various medication-related errors and understand why they occurred, a method was adopted based on a broad ethnographic approach. Participants for this study were selected from various regions of Malappuram district. Crosssectional studies can be thought of as providing a snapshot of the frequency of health related characteristics in a population at a given point in time. The study involves data collection using questionnaire at a single point of time. The data was then analysed for medication-related problems. Using a proper management plan with the help of GP and care giver the patient interventions were carried out. The effectiveness of intervention was then carried out statistically.

Cases were referred by physician, according to the patient needs, and the need for drug counselling review of medication regimen. Pharmacist selects cases for HMR by assessing the patient medications, polypharmacy, risk for medication error, drug interactions etc. After developing a well-defined and validated questionnaire, pharmacist visits patient's home, explains about the study and obtain the patient consent for collecting data. Based on the data collected, prescription inappropriateness like overdose, under dose, medication error, therapeutic duplication etc. are discussed with the physician and management plan are designed. The management plan developed for individual patient was discussed with the patient and caregiver and were then implemented with certain modifications. As an additional service for better patient medication management medication list was provided which was designed using US FDA Medication Card (My medication record). and the data were collected during the study was documented for further analysis. The sample size was calculated as 85 to provide sufficient precision to allow a meaningful estimate of the prevalence of medication errors, and to provide future researchers with sufficient information. The statistical analysis was conducted manually and 'significance' was pre-defined at the $5 \%$ level. Exact binomial confidence intervals were calculated for proportions. Chi-square tests were used to assess differences in error rates between areas. Generalised Estimating Equations were used to model patient level odds of errors, allowing for clustering in homes and using an independence correlation structure. The ethical approval was obtained from Institutional Ethics Comitttee (IEC/ASH/2015/PD/12).

\section{Results}

Data from a total of 85 patients were collected among which 48 were males (56\%) followed by females ( $n=37,44 \%$ ). There is no significant difference in the number of males and females in the sample $\left(\lambda^{2}=1.424\right.$, d. $\left.f=1, p>0.05\right)$.

Out of the 85 patients, majority of the population belongs to the age group 40-59years (47\%) followed by the age group 60-79(39\%) years old. (Mean $\pm \mathrm{SD}=61.50 \pm 14.12$ years). The coefficient of correlation between the age of patients under study and the number of drugs is not significant $(r=0.0909, t=0.831, d f=83$, $\mathrm{p}>0.05)$. Among the various drugs prescribed, the patient had a lack of knowledge in many factors like the name of the drug they are using (34\%), the reason for taking the medication (27\%), and the special instructions (27\%) given during prescribing. (Fig. 1) Drug interaction was the main discrepancy found in majority of the prescriptions, followed by the errors in the name of the medicine. Significant positive correlation exists between total number of drugs and total number of discrepancies $(r=0.4176 \mathrm{t}=4.187, \mathrm{df}=83$ $\mathrm{p}<0.01)$. Since the coefficient of correlation is positive and significant, it indicated that the number of discrepancy increases with the increase in number of drugs (Fig. 2). On HMR, around $32 \%$ of the population experienced ADR on taking the medication $(\lambda 2=11.306, d f=1, p<0.001)$. From the ADR reported, about $89 \%$ of them were Type A reaction (Augmented reaction) and the rest 

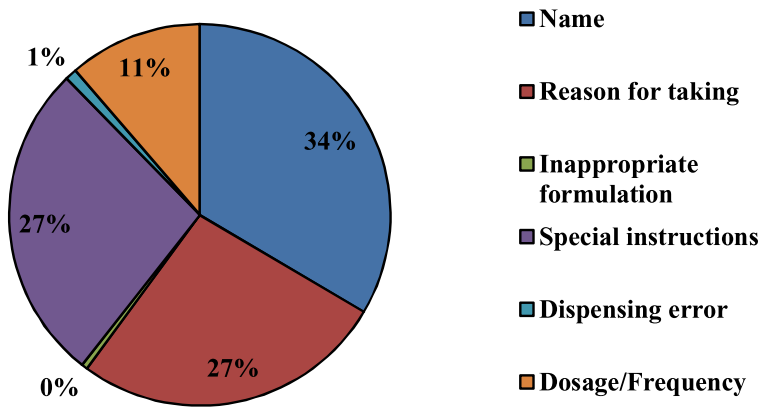

Fig 1. Knowledge gap identified.

were found to be Type $C$ reactions (Continuous reactions) $(\lambda 2=16.333, \mathrm{df}=1 \mathrm{p}<0.001)$. Abdominal pain, gastric irritation and headache ( 3 cases on each symptoms were reported) were the most reactions observed during HMR. Aspirin and Amlodipine were the main drugs found to cause ADR in the patients. Among the patients interviewed, 64\% of them didn't use any OTC drugs along with prescribed drug followed by the use of allopathic (22\%) and ayurvedic $(11 \%)$ medicines in adjuvant with the prescribed $\operatorname{drug}(\lambda 2=75.909, \mathrm{df}=3, \mathrm{p}<0.001)$. Around 60 of the interviewed patients stored multiple drugs in a same container. 52 of the patient's medicines had illegible labels. Errors in 'Multiple Drug Storage' have higher significance than all the rest $(\lambda 2=55.497$, $\mathrm{df}=4, \mathrm{p}<0.001$ ). (Fig. 3 )

Medication adherence was determined using MMAS score (Morisky Medication Adherence Scales). The mean MMAS before and after intervention, differs significantly $(\mathrm{p}<0.05)$. The mean score before intervention is significantly higher than that after intervention.

The main intervention done was medication management (62\%), followed by therapeutic intervention (19\%). $31 \%$ of the patients were provided with patient counselling and there were also given assistance in sorting out the medicines (23\%) and labelling (19\%) them. Number of cases of 'Prescription sorting' is significantly higher than all the rest followed by 'Labelling' and 'Counselling'. (Fig. 4)

\section{Discussion}

HMR play a pivotal role in improving patient care by optimizing prescription appropriateness and improving patient adherence. The results of the study revealed the need for HMR and collaborative approach that can optimize the prescribing and

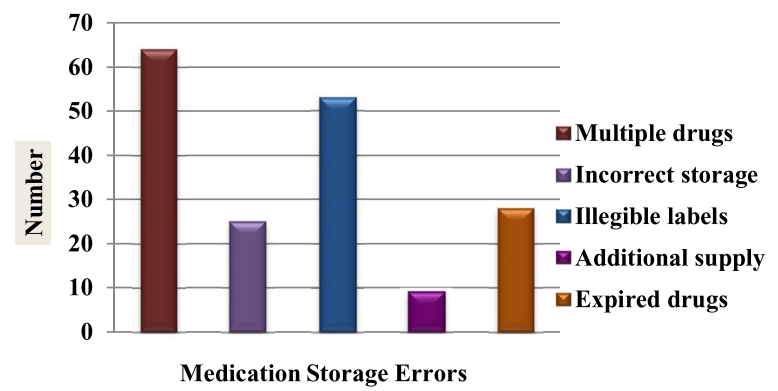

Fig. 3. Storage of medicines.

medication usage in patients. Majority of the population belongs to the age group 40-59 years (47\%) with a mean age of 61.5 years. This may be due to the fact that prescription drug usage mostly included the age group. Age is an independent risk factor contributing to decreased compliance due to age related factors. But the study shows no correlation between age and the increase in number of prescribed drugs. A similar pattern was obtained in studies conducted by Ronald L Castelino et al. ${ }^{5}$, (age of the patients was $75.3 \pm 7.4$ years) and Sorensen et al. ${ }^{6}$ which had an average age of (72.4 years). Educational status plays an influential role in the patient's knowledge of prescribed medications. Among the medication awareness, Knowledge gap is significantly higher in 'Name' compared to others and least in 'Inappropriate formulation' and in 'Dispensing error'. Most of the patients are literate with low proficiency in English language. Most of the patients are aware of the directions, since they are labelled in native language i.e, Malayalam during dispensing. The knowledge gap seemed to be higher as the number of drugs increased and difficulty in remembering the name of drugs. A study conducted by P Kumar et al. ${ }^{7}$, showed poor patient's knowledge of medicines as assessed by recall of name, indications, strength and side effects of medicines. The patients' educational levels and age were found to be negatively associated with knowledge of their medicines. Drug interaction was the main discrepancy found in majority of the prescriptions, followed by the errors in the medicine name. Significant positive correlation exists between total number of drugs and total number of discrepancies. Since the coefficient of correlation is positive and significant, it indicated that the number of discrepancy increases with the increase in number of drugs. Pharmacists play an important role in identifying discrepancies in prescribing and dispensing. Due to increasing workload of physicians, most of the physicians have time constraints leading to improper recording of patient medical

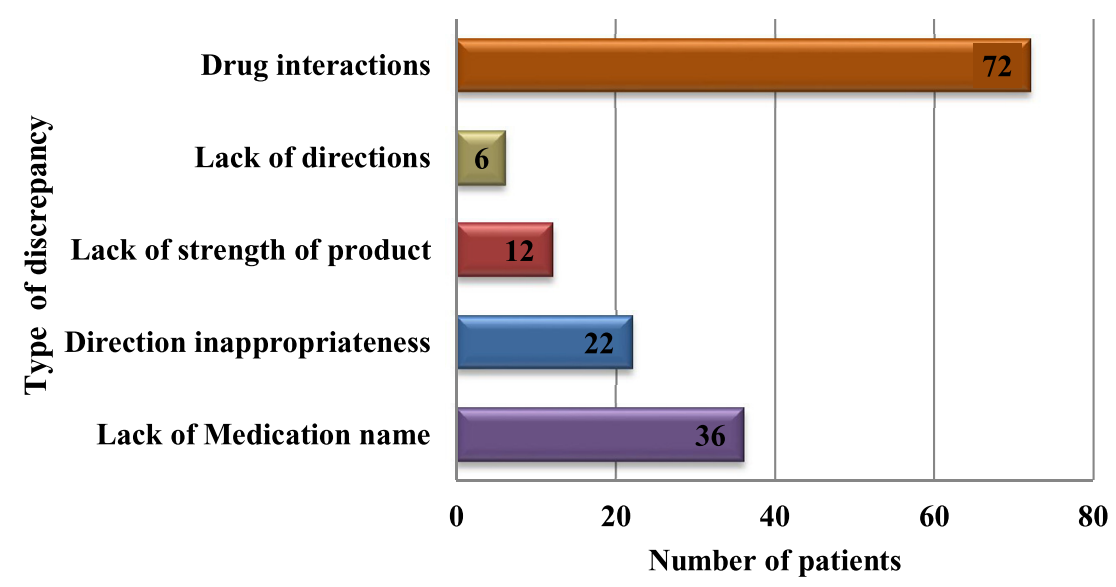

Fig. 2. Types of discrepancy. 


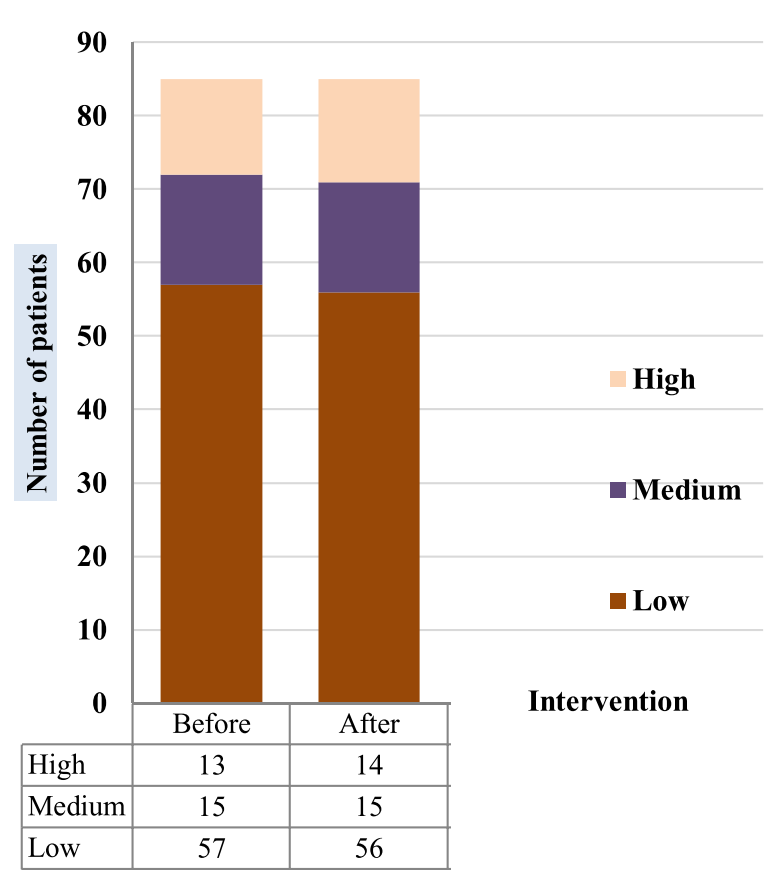

Fig. 4. Medication Adherence.

and medication history. This can lead to untreated indications in patients and most of the patients show a reluctance in informing the physician about medication related adverse events. Nowadays, due to advent of specialized treatment a single patient has multiple prescribers. This has led to prescribing of same medication by multiple prescribers. Also due to absence of patient history, contraindicated drugs are prescribed due to decreased knowledge of inherent patient conditions. This can lead to increased risk of adverse events in patients and decreased patient compliance. ADR can affect the patient's faith in prescriber and treatment and can lead to failure in therapy. During the HMR around 32\% of the population experienced ADR on taking the medication. Many patients are confused about ADR and consider as a disease related difficulty. The patient willingness in reporting adverse events was reduced leading to unidentified ADRs. A considerable amounts of population in the society are suffering from ADR, without being treated. From the ADR reported, about $89 \%$ of them were Type A reaction (Augmented reaction) and the rest were found to be Type $C$ reactions (Continuous reactions). Most of the Type $B$ reactions are identified earlier and during the first dose of therapy. Aspirin and Amlodipine was the main drugs found to cause ADR in the patients undergone Home Medication Review.

OTC drugs can affect the patient's therapy in different ways. Hence there is a need for scrutiny into patient's use of OTC medications. Nowadays, many different OTC drugs are available for a variety of indications. Most of the patients are influenced by peers by promising relief from many of the long term illnesses. But most of the patients are not aware of the possible interactions and harms from the OTC drugs and prescribed drugs. Many drugs used are not even prescribed by physician and are not complying with regulatory requirements. According to the study conducted, $64 \%$ of the patients did not use any OTC drugs apart from prescribed drugs. The various OTC drugs comprise allopathic (22\%) and ayurvedic (11\%) medicines. The patients have less inclination to self medicate and are mostly dependent on physician advice rather than OTC drugs. Storage of drugs plays a pivotal role in patient medication compliance. Around 60 of the interviewed patients stored multiple drugs in a single container which can lead to dose duplication. 52 of the patient's medicines had illegible labels with improper directions leading to decreased drug efficacy and therapeutic failure. Medication adherence was determined using MMAS score (Morisky Medication Adherence Scales). The mean MMAS before and after intervention, differs significantly $(p<0.05)$. The mean score before intervention is significantly higher than that after intervention. This shows that the HMR conducted has significantly helped in increasing patient adherence. The patients showed positive response to counselling and adherence aids as they helped to ease the usage of drugs. The patients were explained about common misconceptions about therapy and various benefits of adhering to medication therapy. Patients were given appropriate information about their diet, medicines, their regimen was simplified and the medication packaging individualized to their particular needs which led to improvement in adherence of patients. Low adherence was significantly higher compared to Medium and High, unveiling that most of the patients have decreased adherence to prescribed medications. Medication counselling was carried out including proper directions. 'Therapeutic Intervention' is significantly higher than providing 'My Medication list' and 'Recommendation to physicians'. 'My Medication list' was provided to patients who had multiple drugs and have difficulty in remembering the directions of medications. In case of prescription errors, the physician or carer was informed. John Papastergiou et al. ${ }^{8}$ indicate that medications were removed from the homes of $58 \%$ of the patients, with expiry of medication being the most common reason for removal of medication. On the other hand, Bell JS et al. ${ }^{9}$ in his study results reveal that pharmacists reported 403 findings and made 360 recommendations for 49 people, with $90 \%$ of recommendations being accepted by the referring general practitioners.

\subsection{Limitations}

The introduction of this study was not an easy task since it is a novel concept. The study requires a collaborative approach with pharmacist and GP. Due to increased workload on physician and due to time constraints the study was affected. The willingness of the patient in providing medication related information and adherence was another hurdle which was faced during the HMR. It was found out that change in medication adherence during reassessment was very less, since it was very difficult to change the routine of the patient

\section{Conclusion}

The study introduced a novel approach of clinical pharmacist for improving patient therapeutic outcome. Home Medication Review was conducted in a series of three patient home visits. The study clearly showed that the subjects were unaware of the HMR service and yet majority were accepting the program. This can be attributed to the lack of clinical pharmacy/community services in India and the perceived role of a pharmacist in the healthcare setup. It is demonstrated through the study that qualified pharmacists can play a major role in improving the appropriateness of prescribing, preventing medication related adverse events. In addition, the improvement in medication adherence following HMR reveals that HMR has the potential to minimize risk of medication inappropriateness and thereby improve patient outcomes. Hence pharmacist in collaboration with General Practitioner can optimize patient medications. A Home medication review greatly decreases the load on tertiary care services, which in India are sadly lacking. 


\section{References}

1. Castelino RL, Bajorek BV, Chen TF. Retrospective evaluation of home medicines review by pharmacists in older australian patients using the medication appropriateness index. Ann Pharmacother. 2010;44:1922-1929.

2. Binti R, Din M. Protocol Medication Therapy Adherence Clinic. Malaysia: Ward \& HMR. Pharmaceutical Services Division, Ministry of Health; 2013:8-10.

3. Chen Tim. The australian model for home medicines review. CPA-MPS Conference. 2007;

4. Elliott RA. Problems with medication use in the elderly: an Australian perspective. J Pharm Pract Res. 2006;36:58-66.
5. Sorensen L. Medication management at home: medication-related risk factors associated with poor health outcomes. Age Ageing. 2005;34:626-632.

6. Kumar PA. clinical pharmacist-based home medication review of geriatric patients. Eur J Hosp Pharm. 2012;19:126.

7. Papastergiou J. Home medication reviews by community pharmacists. Can Pharm J. 2013;146:139-142.

8. Bell JS. Drug-related problems in the community setting: pharmacist's findings and recommendations for people with mental illnesses. Clin Drug Investig. 2006;26:415-425.

9. Furniss Lee. Effects of a pharmacist's medication review in nursing homes, Randomised Controlled Trial. Br J Psychiatry. 2000;176:563-567. 